\title{
Mash Screen: high-throughput sequence containment estimation for genome discovery
}

\author{
Brian D. Ondov ${ }^{1,2^{*}}$ (D), Gabriel J. Starrett ${ }^{3}$, Anna Sappington ${ }^{4}$, Aleksandra Kostic ${ }^{5}$, Sergey Koren ${ }^{1}$ \\ Christopher B. Buck ${ }^{3}$ and Adam M. Phillippy ${ }^{1}$
}

\begin{abstract}
The MinHash algorithm has proven effective for rapidly estimating the resemblance of two genomes or metagenomes. However, this method cannot reliably estimate the containment of a genome within a metagenome. Here, we describe an online algorithm capable of measuring the containment of genomes and proteomes within either assembled or unassembled sequencing read sets. We describe several use cases, including contamination screening and retrospective analysis of metagenomes for novel genome discovery. Using this tool, we provide containment estimates for every NCBI RefSeq genome within every SRA metagenome and demonstrate the identification of a novel polyomavirus species from a public metagenome.
\end{abstract}

Keywords: MinHash, Metagenomics, Sequencing, SRA, Viral Discovery, Polyomavirus

\section{Introduction}

As evolving sequencing technology continues to increase throughput and lower costs, databases of sequenced genomes (e.g., NCBI RefSeq [1]) continue their exponential growth, making searches against them ever more complex [2, 3]. Furthermore, the body of raw sequencing data, NCBI's Sequence Read Archive (SRA) [4], is growing even faster, outpacing our ability to assemble and curate the genomes and metagenomes represented $[5,6]$. These trends have popularized alignment-free analytical methods, typically operating on $k$-mers, which are short $(\approx 21 \mathrm{bp}$ ), overlapping subsequences of the genome for some length $k$. Our prior work in this area introduced Mash [7], which uses the MinHash dimensionalityreduction technique [8] to compress $k$-mer sets of whole genomes to sketches of just several hundred or thousand values, enabling extremely fast estimations of genomic or metagenomic distances. Though useful for isolated genomes or high-level metagenomic correlation, Mash is

*Correspondence: brian.ondov@nih.gov

${ }^{1}$ Genome Informatics section, National Human Genome Research Institute, Bethesda, MD, USA

2Department of Computer Science, University of Maryland College Park, College Park, MD, USA

Full list of author information is available at the end of the article ill-suited for analyzing the constituents of a metagenomic mixture. This is because typical applications of MinHashing approximate resemblance rather than containment. In this work, we address the containment problem and present a new method, Mash Screen, for measuring how well a genome or proteome is represented within a metagenome. This has applications ranging from quick contamination screens to the tracking of specific bacterial and viral strains across all available metagenomes.

Though originally defined in terms of documents and words, here we will consider the resemblance and containment of biological sequences and their constituent $k$-mers. For two genomes $a$ and $b$, their resemblance is defined as the Jaccard index $j$ between their $k$-mer sets $A$ and $B, j_{k}(a, b)=\frac{|A \cap B|}{|A \cup B|}$. Thus, resemblance is sensitive to differential sequence lengths. Consider a single genome $a$ and a much larger metagenome $b$. The resemblance between $a$ and $b$ is expected to be low, even if $b$ wholly contains $a$, because $b$ will likely contain many $k$-mers not present in $a$, making the union of $A$ and $B$ larger than the intersection. Resemblance ranges from 0 to 1 , is symmetric, and $1-j$ is a metric. In contrast, the containment index $c$ is asymmetric and measures the extent to which one genome is contained in another, $c_{k}(a, b)=\frac{|A \cap B|}{|A|}$. If all 
the $k$-mers of $A$ are contained in $B$, regardless of how many other $k$-mers $B$ contains, the containment index will equal 1 . Because genome sequences can be very long, we seek to efficiently approximate $j_{k}(a, b)$ and $c_{k}(a, b)$ by considering only a small fraction of the total $k$-mers.

For estimating resemblance, Mash uses a "bottom sketch" strategy as originally proposed by Broder [8]. More efficient techniques for estimating resemblance have since emerged [9-12], but bottom sketching is elegant in its simplicity. In short, all $k$-mers from a genome $A$ are passed through a single hash function $h$, but only the smallest $m$ hash values are stored as the sketch $S(A)$, where $|S(A)|<<|A|$. Because the probability that
$S(A)$ and $S(B)$ share a minimum value is related to the Jaccard index of $A$ and $B$, the resemblance of the full $k$-mer sets $A$ and $B$ can be quickly approximated from the comparatively smaller sketches $S(A)$ and $S(B)$. Mash further converts resemblance to the Mash distance, which is an estimate of mutational distance between the two sequences.

While the difference in formulation between resemblance and containment is small, different strategies are required to accurately approximate them. In contrast to resemblance, which can be reliably estimated using fixed-size sketches, estimating containment requires a sketch size proportional to the genome size (Fig. 1).

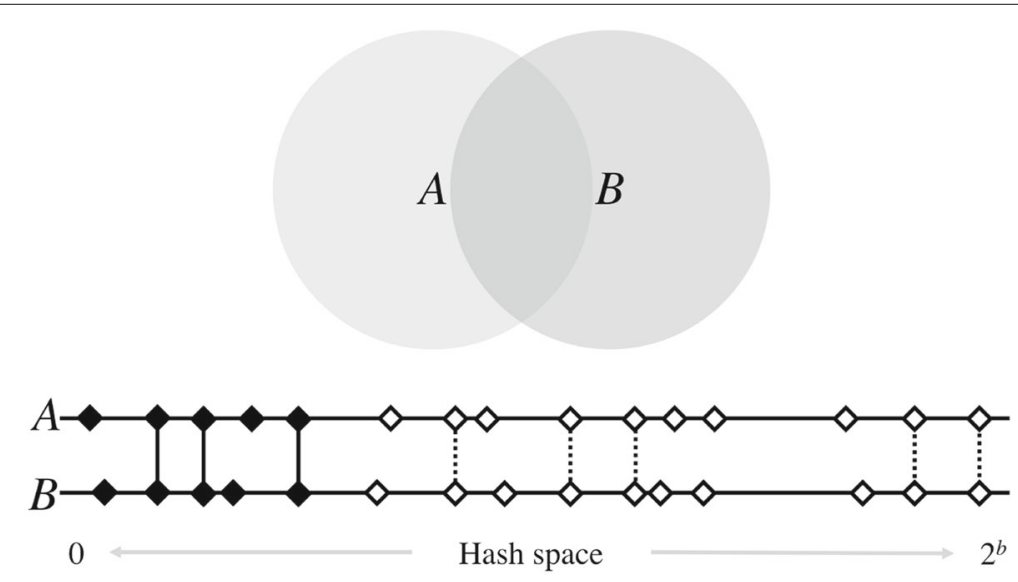

(a)

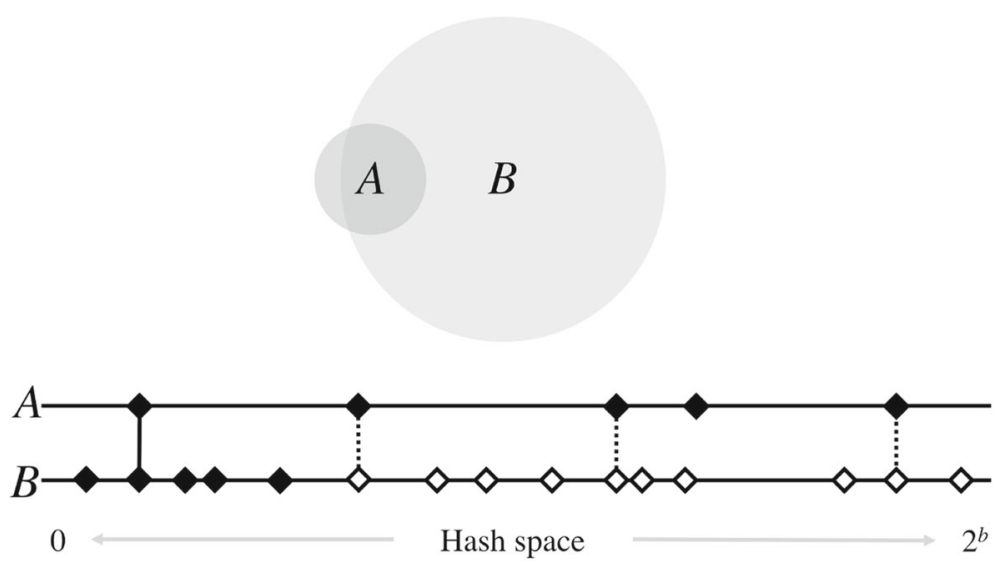

(b)

Fig. 1 High-level diagram of set operations with MinHash. The size of the shaded circles represents $k$-mer set sizes, while the area of their overlap represents the cardinality of their intersections. Below each pair of circles is a diagram of the MinHash resemblance estimation for a sketch size of 5. Horizontal lines represent the space of possible hash values, of which there are $2^{b}$, where $b$ is the number of bits used for hashing. Here, diamonds are hashes of the $k$-mers in sets $A$ and $B$, and black shading indicates the smallest 5 hashes in each. Vertical lines indicate matching hashes and are solid only if both hashes are in the bottom sketch of their respective set. In $\mathbf{a}$, genomes of similar sizes are well-suited for resemblance estimation, since their hashes are similarly distributed across the hash space. Matching hashes are usually both in, or both out, of the bottom 5 . However, if the genomes are of very different sizes, as in $\mathbf{b}$, the larger genome will saturate the space more densely. This causes a higher fraction of matching hashes to be contained in only the sketch of the smaller set, underestimating the containment of $A$ in $B$. Thus, all hashes of $B$ must be considered to accurately estimate the containment of $A$ 
To accomplish this, Broder originally proposed using a modulo function for containment sketching, which produces proportionally sized sketches. However, the modulo sketch has the disadvantage of requiring a very large sketch size in order to achieve acceptable error bounds when searching for small contained elements, such as viruses. To bound the size of this data structure, Bloom filters have been suggested as a replacement for modulus sketches when estimating containment [13]. In the Bloom filter scheme, $|A \cap B|$ is estimated using a MinHash sketch of $A$ and a Bloom filter of $B$. For the specific application of searching transcriptomes, Bloom trees [14] and Counting Quotient Filters [15] have been proposed. Additionally, Bloom filter matrices have been used for searching within unassembled genome isolates [16].

These prior containment estimation methods have all sought to compress and/or index a large collection of sequencing reads, with the goal of enabling rapid sequence search across them (e.g., given a reference database of transcripts, isolates, or metagenomes, identify all those containing some query sequence). This is an important problem, but in this paper, we consider the converse: given a reference database of genomes, identify all those contained in some query metagenome. We would also like to estimate, without assembly, the similarity between the reference genomes and those contained in the metagenome.

To address this problem, we propose the concept of a screen, in which we rapidly test a database of many genomes for their containment within a larger, unassembled metagenome (Fig. 2). For each reference genome, Mash Screen computes a containment score that measures the similarity of the reference genome to a sequence contained within the metagenome. This enables routine tasks such as quick contamination screening, the selection of appropriate reference genomes for mapping-based analyses, and efficient screening of entire metagenomic databases for tracking individual species and strains across all samples. For the latter case, we have computed the containment of every NCBI RefSeq genome within every NCBI SRA metagenome and have made these results available for download and query. To demonstrate the potential utility of this dataset, we describe the identification and assembly of a novel polyomavirus species from a public SRA metagenome.

\section{Results}

\section{Correlation between Mash distance and containment estimates}

For the $k$-mers of a given reference genome $a$, a related genome $a^{\prime}$ that is contained within a metagenomic read set $b$ should, by design, have a Mash containment score, $M_{c}(a, b)$ (see "Mathematical foundations"), that is inversely correlated with the Mash distance $M_{d}\left(a, b^{\prime}\right)$. Ideally, $M_{c}(a, b)=1-M_{d}\left(a, a^{\prime}\right)$. However, because the metagenome may contain $k$-mers matching $a$ that are not from $a^{\prime}$, and the different formulations of the Mash distance and containment, the correlation is imperfect.

To test how well the Mash containment score correlates with the Mash distance, we analyzed metagenomic reads, both real and simulated, from a mock community of bacteria and archaea with known constituents [17], referred to here as the Shakya dataset. For expected scores, we used all RefSeq genomes close in size to Shakya

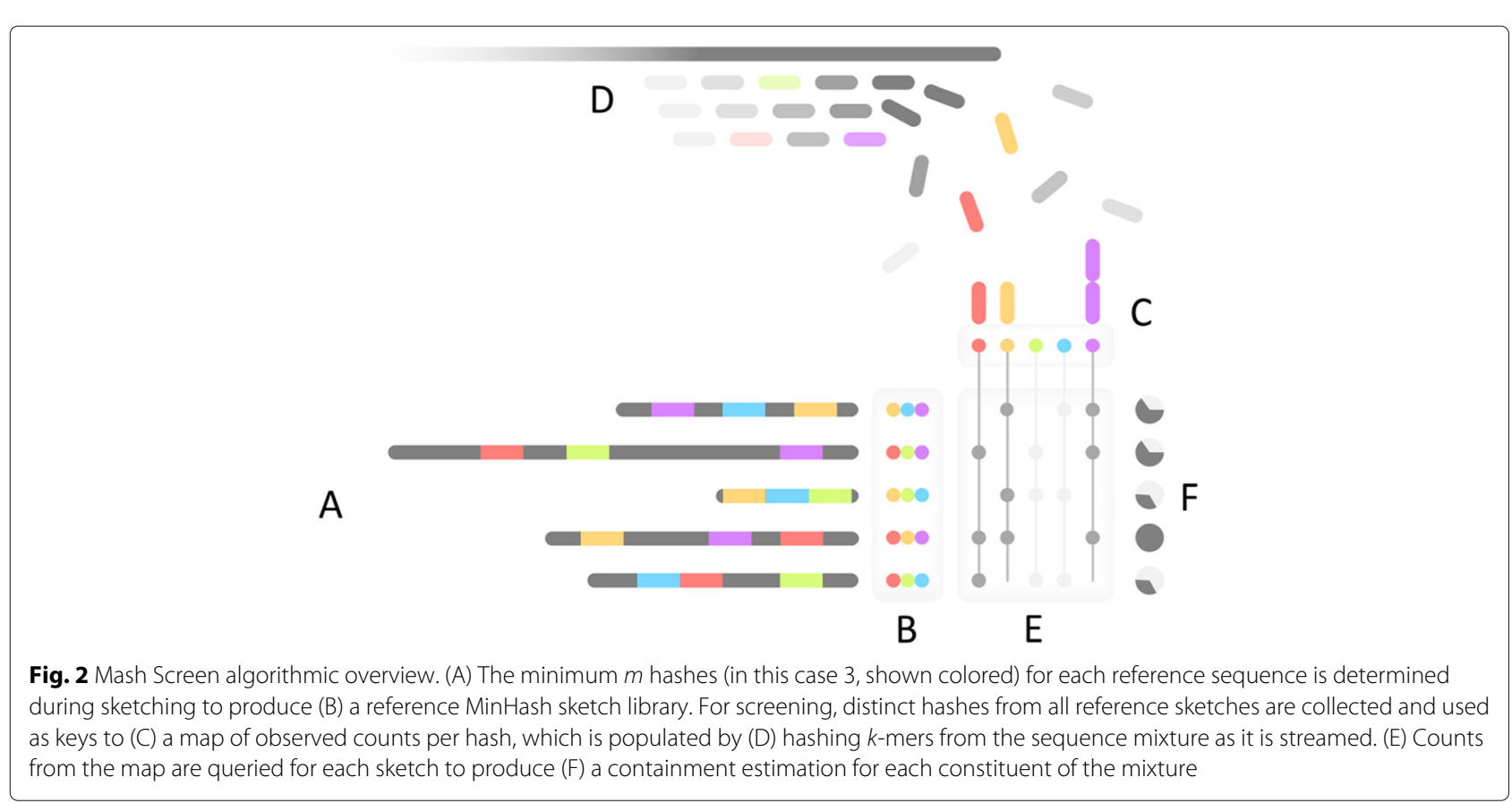


constituents (between 100K and 20M nucleotides, effectively excluding viruses and eukaryotes) and computed high-accuracy (sketch size 100,000), whole-genome Mash distances between each of these genomes and their nearest Shakya constituent. These distances were compared to Mash containment scores for the same genomes, obtained by screening raw metagenomic reads against RefSeq. Screening the 11.1 gigabases of Illumina reads against RefSeq (compressed from $1.14 \mathrm{~Tb}$ to a $1.2 \mathrm{~Gb}$ sketch) for this analysis took $61 \mathrm{CPU}$ minutes, using a peak of $11 \mathrm{~Gb}$ of memory (system details in "Correlation between Mash distance and containment estimates"). Though not directly comparable to the indexing methods mentioned above, which have both different approaches and applications, the database compression of Mash $(950 \times$ in this case) is of a similar scale to that reported by Mantis [15] $(250 \times)$ or BIGSI [16] $(113 \times)$. Memory requirements of these tools tend to closely track the size of these indexes, and this is true of Mash Screen as well.

Mash containment scores showed good correlation for Mash distances less than $0.2\left(r^{2}=0.99\right)$, but tended to overestimate Mash distances greater than $0.2\left(r^{2}=0.43\right.$ for all points with nonzero containment scores, Fig. 3a). First, like Mash distance estimates, the expected error of the containment estimates increases for more diverged sequences. Second, when estimating containment, $k$-mers from any genome in the mixture are permitted to match the reference, whereas the Mash distance is restricted to matching only $k$-mers from the genome being compared. Figure 3 (left) also highlights a number of outliers that sit well off the diagonal. Points circled in red represent RefSeq genomes that do not share high similarity to the expected constituent genomes, yet have high containment scores when compared to the metagenome. This indicates suspected contamination in the Shakya mock community.

Escherichia coli and Proteiniclasticum ruminis contamination had been previously detected in this mock community by Awad et al. [18], and so we speculated that this was causing the discrepancy between Mash distance and containment estimates. Consistent with this explanation, simulating reads from only the listed constituents improved overall correlation for nonzero containment scores to $r^{2}=$ 0.73 (Fig. 3, right). Further investigation of the outlier points revealed additional Propionibacterium acnes and Streptococcus parasanguinis contamination that was not identified by the prior studies. Mapping all metagenomic reads to these reference genomes confirmed the presence of the four contaminating species or their near relatives (Table 1). After filtering points arising from these contaminants, correlation of the real dataset was similar to that of the simulated dataset $\left(r^{2}=0.72\right)$. Also notable are points closer to the diagonal, but still in distinct clusters, highlighted in magenta and orange in Fig. 3 (left). These represent two distinct clades of $F$. nucleatum, a gram-negative commensal of the human oral cavity that has well-delineated subspecies [19]. The expected reference strain, ATCC 25586, was noted by Awad et al. to be present in low abundance, which is likely what is causing the Mash containment to be lower than expected for this and similar strains (below the diagonal, circled

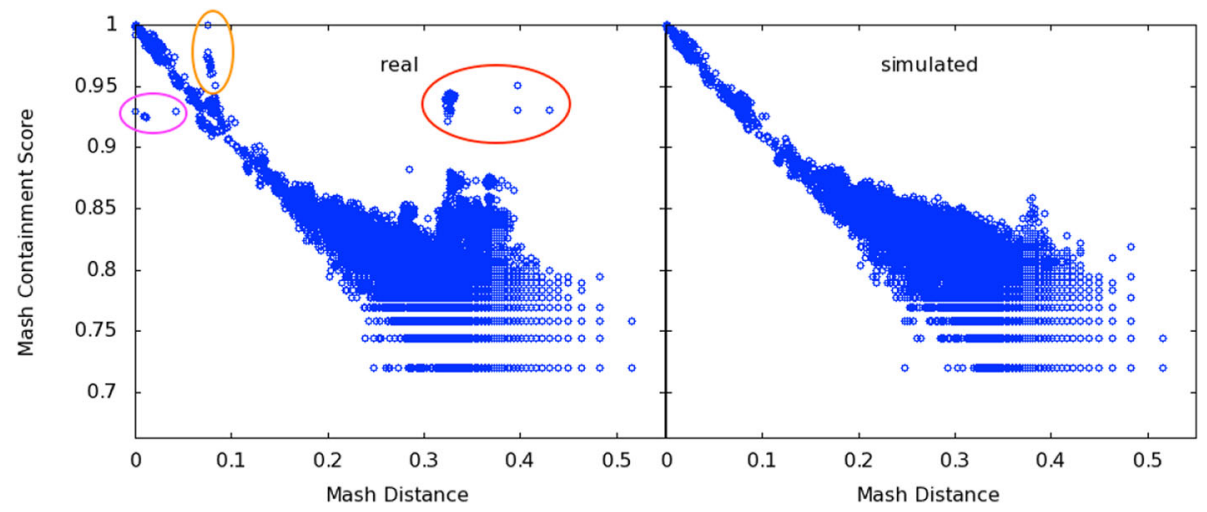

Fig. 3 Correlation of Mash containment scores with pairwise Mash distances. Points represent RefSeq genomes with sizes between 100K and 20M The $x$-axis is the Mash Distance to the nearest genome in the Shakya synthetic metagenome, which serves as an expected containment score. For the $y$-axis, raw reads from this metagenome were run through Mash Screen (sketch size 1000) to obtain an actual containment score for each of the genomes. Left, real Illumina reads sequenced from the mock community (SRR606249). The area circled in red highlights RefSeq genomes with higher than expected containment scores caused by contamination of the sequencing run. This contamination was independently confirmed via read mapping, revealing the presence of at least four additional species. Circled in magenta and orange are two clades of $F$. nucleatum that are consistent with low abundance of the reference strain (magenta) and intra-species contamination (orange), both of which have been previously described for this dataset. Right, simulating reads from only the known constituents corrects the outliers and yields the expected correlation. Not shown: 1645 points in $(0.28 \leq x \leq 0.52, y=0)$ (left plot) and 356 points in $(0.32 \leq x \leq 0.52, y=0)$ (right plot), representing the limits of sensitivity of the default sketch size (1000) used for the $y$-axis, compared the the higher sensitivity used for the $x$-axis (sketch size of 100,000). No points lie to the right of the plot areas 
Table 1 Identified contaminants in the Shakya dataset measured by mapped reads and Mash Screen

\begin{tabular}{llllll}
\hline Organism & Reads & Coverage & Identity & Score & p value \\
\hline $\begin{array}{l}\text { Propionibacterium } \\
\text { acnes HL072PA1 }\end{array}$ & 36,222 & $7.29 \%$ & $96.36 \%$ & 0.874 & $3.49 \mathrm{e}-136$ \\
$\begin{array}{l}\text { Escherichia coli } \\
\text { strain 2014C-3250 }\end{array}$ & 59,744 & $4.99 \%$ & $95.16 \%$ & 0.837 & $7.32 \mathrm{e}-47$ \\
$\begin{array}{l}\text { Proteiniclasticum } \\
\text { ruminis DSM 24773 }\end{array}$ & 751,538 & $76.57 \%$ & $91.83 \%$ & 0.930 & 0 \\
$\begin{array}{l}\text { Streptococcus } \\
\text { parasanguinis }\end{array}$ & 74,807 & $57.50 \%$ & $95.84 \%$ & 0.942 & 0 \\
strain C1A & & & & & \\
\hline
\end{tabular}

Organism refers to the closest strain in RefSeq to the suspected contaminant, based on Mash Screen distance. Reads refers to the number of reads mapped to those genomes. Coverage refers to the amount of each genome that was covered by mapped reads. Identity refers to the average identity of the covered portions based on naive consensus from the pileups. Score and $P$ value are results from Mash Screen

in magenta). The strains above the diagonal (circled in orange), however, represent Fusobacterium nucleatum subspecies (polymorphum and vincentii) that Awad et al. suggested could be intraspecies contaminants. The results of this experiment are thus widely in concordance with prior, independent research, lending validation to both the past findings and to our new method.

\section{What is in my sequencing run?}

As illustrated above, Mash Screen can discover unexpected organisms in sequence mixtures. This can be useful for checking any sequencing run for accidental contamination. While exploring containment results for a number of SRA datasets, we identified a number of potential contamination events. As an example, SRA run ERR024951 is a 1.1-Gb short-read dataset labeled as Salmonella enterica subsp. enterica serovar Typhimurium. However, analysis with Mash Screen also indicated the presence of Klebsiella pneumoniae, with strain k1037 (GCF_900086185.1) being the closest hit. We verified the presence of the contaminant by mapping all reads to both Salmonella enterica and Klebsiella pneumoniae reference genomes. This revealed that the majority of reads in this sample were in fact from Klebsiella pneumoniae (Table 2). The metadata on this particular dataset notes that it is part of a multiplexed sequencing run, suggestive of a possible contamination source. Because Mash Screen is extremely fast to run, it can be used as part of a standard sequencing workflow to catch such events before data is submitted to the public archives.

In the above examples, we show how Mash Screen can identify a "shortlist" of genomes that are likely to be in a sequencing run. This can be generalized to the problem of metagenomic classification, where reference databases are often too large to allow traditional sequence analysis techniques such as read mapping. While not a classifier in its own right, Mash Screen can help address this
Table 2 Read mapping to confirm contamination of Klebsiella pneumoniae in a run, ERR024951, labeled as Salmonella enterica

\begin{tabular}{llllll}
\hline Organism & Reads & Coverage & Identity & Score & $p$ value \\
\hline $\begin{array}{l}\text { Salmonella } \\
\text { enterica subsp. }\end{array}$ & $1,962,751$ & $88.91 \%$ & $98.64 \%$ & 1 & 0 \\
$\begin{array}{l}\text { enterica serovar } \\
\text { Typhi str. CT18 }\end{array}$ & & & & & \\
$\begin{array}{l}\text { Klebsiella } \\
\text { pneumoniae } \\
\text { strain k1037 }\end{array}$ & $10,726,719$ & $98.76 \%$ & $99.96 \%$ & 0.999 & 0 \\
\hline
\end{tabular}

Organism refers to either the labeled genome or the contaminant. Reads refers to the number of reads mapped to those genomes when both were provided as references in tandem. Coverage refers to the amount of each genome that was covered by the mapped reads. Identity refers to the average identity of the covered portions based on naive consensus from the pileups. Score and $P$ value are results from Mash Screen

problem by quickly filtering out reference genomes that are not well contained by the query mixture. This can drastically reduce the number of reference sequences necessary for good mapping and/or classification results. To test this application, we compared the known truth of the Shakya metagenome to the results of screening the reads for containment of RefSeq genomes. The filtered genomes amount to a significant size reduction in terms of the fraction of nucleotides represented, but still encompassed the vast majority of the known constituent genomes (Table 3). Note that a threshold of 1 (perfect containment) leaves many genomes out because Mash Screen is sensitive to both identity and completeness, and it has been observed that coverage of some genomes in this sample is low [18]. This experiment is thus a good illustration of the tradeoff between database size and classification sensitivity that may be necessary. However, in this case, pre-screening to a Mash containment of 0.9 retains all correct reference genomes while significantly reducing the database size, and tightening the threshold to 0.99 retains $56 / 58$ of

Table 3 Potential reduction in classification database size by pre-screening, illustrated using constituents of the Shakya metagenome

\begin{tabular}{lllll}
\hline Containment $(\geq)$ & Positives & Positives (\%) & $\begin{array}{l}\text { Database } \\
\text { genomes }\end{array}$ & $\begin{array}{l}\text { Database } \\
\text { gigabases }\end{array}$ \\
\hline 0 & $58 / 58$ & 100 & 83,327 & 330 \\
0.9 & $58 / 58$ & 100 & 1585 & 5.76 \\
0.99 & $56 / 58$ & 96 & 227 & 0.878 \\
0.999 & $53 / 58$ & 91 & 76 & 0.237 \\
0.9999 & $43 / 58$ & 74 & 58 & 0.182
\end{tabular}

Containment refers to the threshold of the Mash containment score used to filter the genomes in the database, with 0 meaning no filtering and 0.9 meaning filter all genomes with a containment of less than $90 \%$. Positives refers to how many of the known constituents had scores above that threshold, and thus would pass pre-screening ( 6 of the 64 constituents were not included because they have since been removed from RefSeq and thus were not in the reference database). Database genomes refers to the total number of genomes in the database with scores above the threshold, and Database gigabases refers to the total number of bases in those genomes 
the correct genomes while reducing the database size an additional order of magnitude. Thus, Mash Screen can be helpful for recruiting a suitable set of reference genomes for metagenomic read mapping.

Estimating proteome containment with a translated screen Mash Screen introduces support for an amino acid alphabet, including 6-frame translation of the input reads. This allows for protein sketching and proteome containment estimation directly from metagenomic sequencing reads, which can be helpful when searching for divergent genes or genomes using short reads. To demonstrate that Mash's $k$-mer-based approach can effectively measure protein similarity, we compared Mash Screen to the commonly used translated alignment program DIAMOND [20]. For a reference set, we began with reported protein fragments from two samples of the Human Microbiome Project [21], SRS015937 and SRS020263, and recruited their best hits to full-length proteins in the NCBI $\mathrm{nr}$ database, resulting in 28,334 protein sequences. Sequence reads from only one sample, SRS020263, were then mapped with DIAMOND to this combined set of proteins to ensure a broad range of alignment scores. Note that Mash and DIAMOND are designed for different tasks. Mash estimates global similarity, whereas DIAMOND computes local alignments. To estimate global similarity from DIAMOND alignments, a consensus residue was computed at each aligned reference position by simple majority, and the total number of matches was divided by the reference protein length to estimate global similarity, accounting for both similarity and coverage. By default, and as run, DIAMOND reports the single best HSP (i.e., mapping) for up to 25 reference sequences for each read. The consensus scores thus describe how well each protein is represented in the reads, without assigning each read exclusively to one protein, analogously to Mash Screen. The consensus scores were compared to the Mash containment scores computed by screening the sequencing reads against a sketch library consisting of the same reference proteins. Overall correlation between the two scores was $r^{2}=0.64$, and the relationship remained linear even for proteins with low coverage (Fig. 4). As was seen in the above comparisons to the Mash distance, the Mash containment score tended to overestimate the pairwise DIAMOND scores, especially with lower scores, but in a fairly consistent way.

Sequence sketching confers its biggest advantage when reference sequences are long. Thus, in the case of individual proteins, Mash Screen is only slightly more efficient than a mapping-based tool such as DIAMOND (41 versus

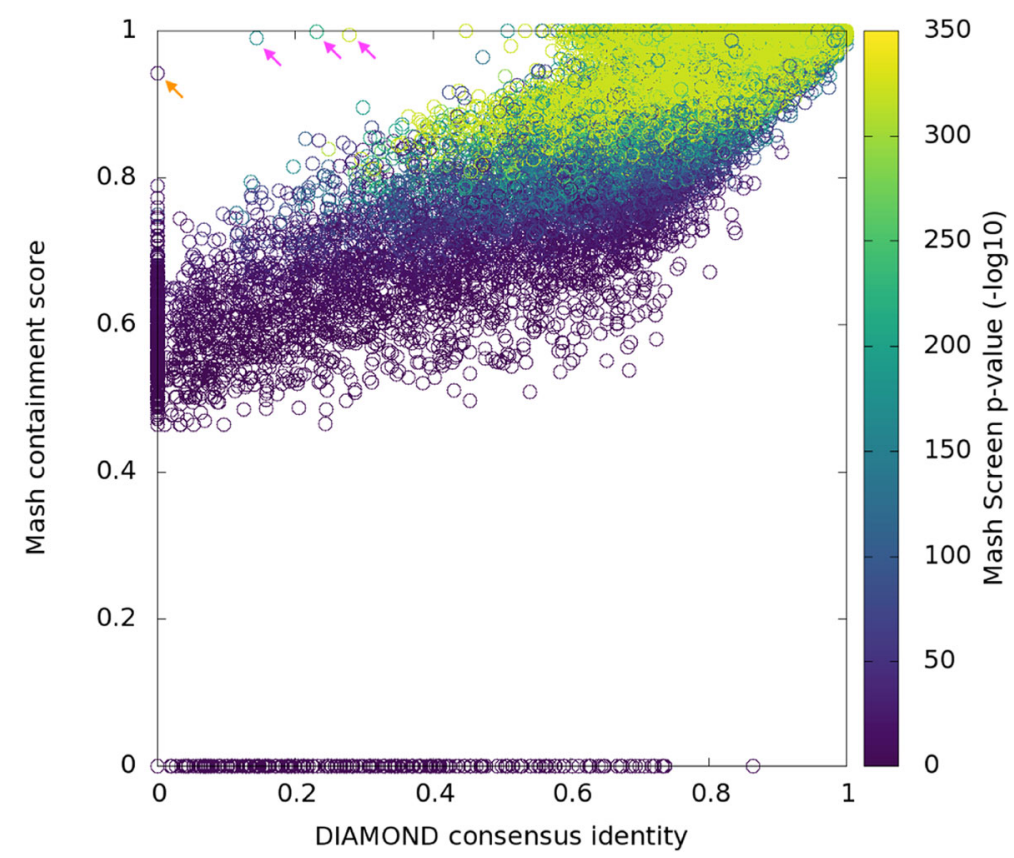

Fig. 4 Correlation of Mash Screen containment scores with DIAMOND read mapping identity, each using six-frame translations to compare nucleotide reads to protein reference sequences. Each point represents a gene from the recruited set chosen for the experiment. Position on the $x$-axis corresponds to global alignment identity estimated from mapping, while position on the $y$-axis represents the containment score for the same protein as reported by Mash Screen. Coloring represents the Mash Screen $p$ value. The points indicated by arrows have legitimate alignments that were not picked up by DIAMOND with its most sensitive settings; in orange a short (20aa) sequence (AMP55843.1) and in magenta sequences with low-complexity regions (ESV13988.1, EYQ74458.1,WP_002468660.1) that were not aligned despite disabling the low-complexity filter. Points along the $x$-axis represent the limits of Mash Screen's sensitivity. For these genes, mismatches were common enough, and evenly distributed enough, to change all $k$-mers indexed by Mash Screen, which in this case were 9 amino acids long 


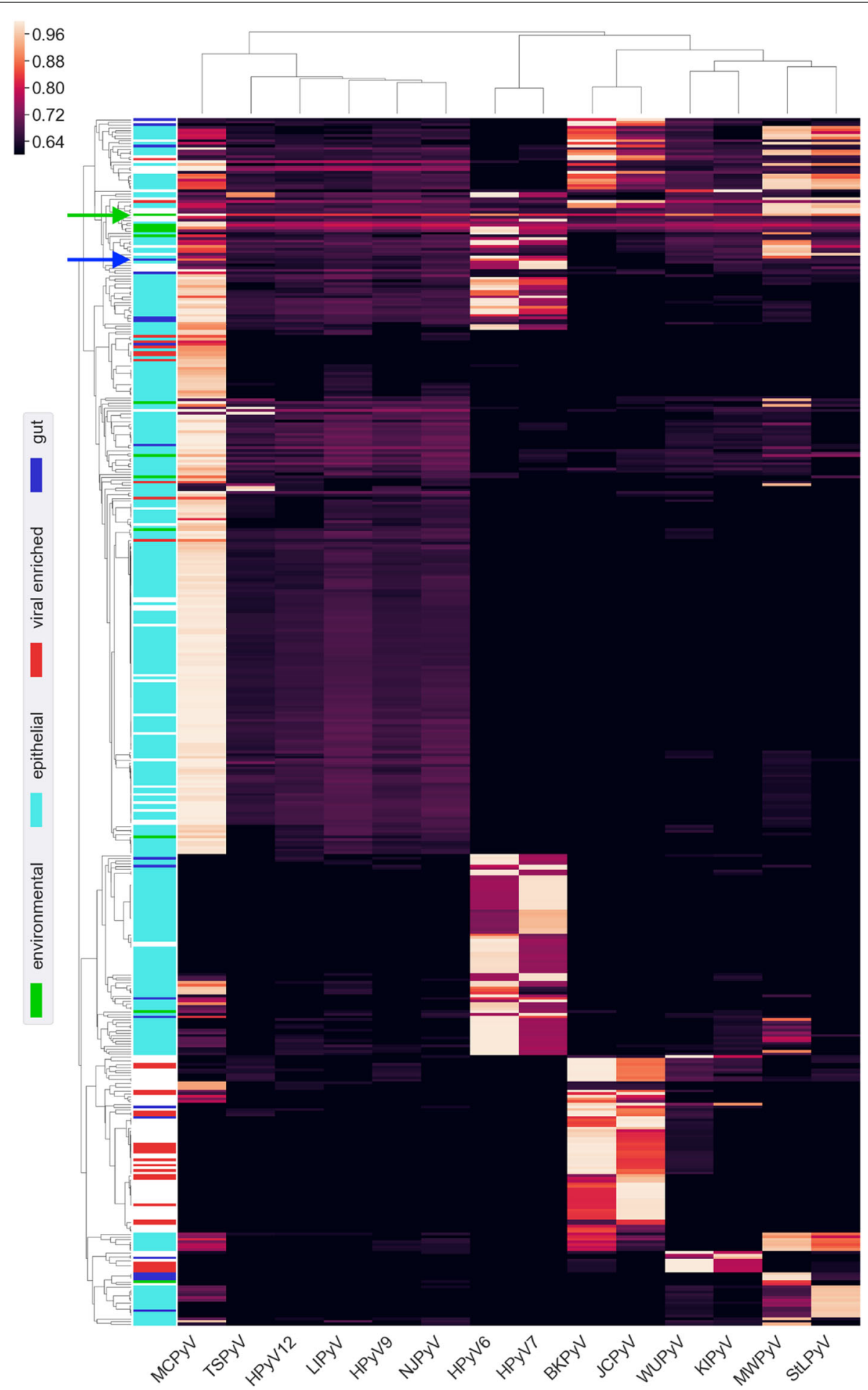

Fig. 5 Metagenomic SRA runs with hits to human polyomaviruses. Hierarchical clustering was applied to both axes, and, where possible, descriptions from the organism metadata field were used to label various categories, depicted in the colored bands on the left. The vast majority of PyV-positive epithelial samples (cyan) are skin metagenomes from an eczema study (BioProject PRJNA46333). The blue arrow indicates the fecal sample in which QPyV was initially detected (SRR2565980). As an example environmental sample with strong hits to multiple human polyomaviruses, the green arrow indicates a money metagenome (SRR5256705) 
121 CPU hours in the above experiment), because the resulting sketches are nearly as large as the original proteins. Instead, Mash Screen is designed for measuring the containment of whole genomes and proteomes within many metagenomes. Because screening a whole proteome sketch requires no additional space or runtime compared to screening a single protein, Mash scales extremely well in this case, whereas using a mapping-based tool would be infeasible. The below sections illustrate how this scalability can be used to process the entirety of the NCBI Sequence Read Archive to make new genomic discoveries.

\section{Screening all metagenomes in the Sequence Read Archive} Mash Screen was designed to process the enormous collection of metagenomic data available in the public sequence archives, and scales linearly with the size of the input-requiring only slightly more time than would be required to simply read a FASTQ file. We leveraged this efficiency to process the entire metagenomic cata$\log$ of the NCBI Sequence Read Archive, and compute a containment score for every genome and plasmid in NCBI RefSeq versus every metagenome in the SRA. At the time of this analysis, there were 144,909 metagenomic sequencing runs (totalling approx. $4 \mathrm{~PB}$ of files) and 103,346 RefSeq genomes and plasmids (totalling 1.14 TB) in NCBI. Note that during analysis, a small number of runs (between 1 and 2\%) could not be accessed due to removal from the database, access restrictions, or repeated failed server requests (lists of the queried runs are provided with online data). The resulting table, although sparse, represents a total of approx. 15 billion containment estimates. Total runtime was approx. 500,000 CPU hours (approx. $1,000,000$ for six-frame translated), which is feasible to recompute for each major RefSeq release with parallelization. Comparatively, appending additional SRA runs to the full table is trivial and requires a cost of just $8 \mathrm{CPU}$ minutes per $1 \mathrm{Gbp}$ of sequence (16 CPU minutes for six-frame) and using 10-15 GB of memory.

Here, we make available the results of the RefSeq-SRA containment screen, consisting of all data available as of August 2018. To limit the size of these tables, we provide the results filtered in two ways: one table contains matches above a 0.95 containment threshold, while another relaxes this threshold to 0.80 but requires $3 \times$ median $k$-mer multiplicity (that is, at least half of the $k$-mers in a given RefSeq sketch must be observed 3 or more times in the SRA run; this value is output by Mash Screen). The former is useful for finding runs that contain a specific target, while the latter is useful for discovering novel sequences, as demonstrated in the following section. Screens were run using both untranslated nucleotide and translated protein screens, resulting in a total of four tables, available in online data.

\section{Global distribution of human polyomavirus species in the SRA}

Polyomaviruses are chronically shed from epithelial surfaces and are known to be common constituents of clinical and environmental samples. Although recent sero-epidemiological studies have confirmed that most humans are immunologically exposed to human polyomaviruses HPyV 1 through 11, seroreactivity against HPyV12, New Jersey polyomavirus (NJPyV), and LyonIARC polyomavirus (LIPyV) is puzzlingly rare [22, 23]. It is thought that polyomaviruses generally co-speciate with their hosts and, accordingly, polyomavirus phylogeny tends to recapitulate the phylogenetic relationships of host animals [24]. Application of this principle led to the recent suggestion that HPyV12 may have been a laboratory contaminant that ultimately originated from shrew specimens [25]. Similarly, LIPyV was originally detected by PCR in human saliva but shows low seroreactivity in the general population [22], suggesting an external source for the original detection (e.g., household pets).

We selected all SRA metagenome runs with a proteome containment score $\geq 0.8$, a median $k$-mer multiplicity $\geq 3$ (as for the table in "Screening all metagenomes in the Sequence Read Archive"), and a $p$ value $\leq 1 \times 10^{-100}$ for at least one human-associated polyomavirus. Fig. 5 shows containment scores for the 456 (of approx. 143,000 total) runs meeting these criteria. The results show that most known human-tropic polyomaviruses were contained in multiple metagenomes. In contrast, $\mathrm{HPyV} 12, \mathrm{NJPyV}$ and LIPyV were not detected in any metagenomes. The result adds complementary support for serological and phylogenetic evidence that human infection with HPyV12, NJPyV, and LIPYV is rare or nonexistent.

NJPyV was initially detected in an immunosuppressed woman who was exposed to flood waters in the wake of Hurricane Sandy [26]. The virus was detected by in situ hybridization in myositic and cutaneous lesions, conclusively demonstrating productive infection of the patient. In contrast to HPyV12 and LIPyV, NJPyV shows close (70-80\%) nucleotide similarity to polyomaviruses found in old world primates. A possible explanation for these observations is that NJPyV is a bona fide human-tropic virus but its prevalence is extremely low.

To search for additional examples of the hypothetical NJPyV category, we screened for SRA runs containing good (but not perfect) hits to known polyomavirus species (Fig. 5). One identified run was a metagenomic analysis of human fecal samples from an 85-year-old hospital patient in Montreal, Canada (SRR2565980). The top polyomavirus hits for this run were HPyV6 and HPyV7, with $k$-mer multiplicities of 2 and 3 , and proteome containment scores of 0.88 and 0.82 , respectively. Lack of a containment score $\geq 0.90$ to any known polyomavirus was interpreted as evidence for a potentially novel virus. 
All reads from this metagenome were assembled with metaSPAdes, revealing a nearly complete polyomavirus related to HPyV6 and $\mathrm{HPyV} 7$, which was manually finished to recover the complete genome. This new virus, for which we suggest the name Quebec polyomavirus (QPyV, GenBank BK010702), is 80\% identical to HPyV7 and 67\% identical to HPyV6 at the nucleotide level. As additional evidence for the new virus, another run (SRR2565981), representing an additional time point for the same patient, also had Mash containment scores passing the threshold $(0.82$ and 0.84 , with median $k$-mer multiplicity 3 and 6, respectively, for HPyV6 and HPyV7). Investigation of a third time point from the same patient (SRR2565982) also revealed 4 reads mapping to the new QPyV assembly, which was below the Mash detection threshold. Although the high degree of similarity of QPyV to HPyV6 and HPyV7 strongly suggests that it is a primate-tropic polyomavirus, QPyV was only detected in fecal samples from a single study of hospital patients. It remains unclear whether QPyV (or NJPyV) should be thought of as a truly human-tropic polyomavirus.

\section{Discussion Limitations}

Mash Screen quickly measures the containment of a set of reference sequences within a metagenomic mixture. Note, however, that each of the reference sequences is treated independently, without consideration for redundancy or overlap among others. For example, many different strains of a given species would be expected to have high containment scores even if only one strain is present in the mixture. These measurements thus do not, in themselves, estimate the composition of a metagenome but rather measure how well each reference genome is contained within the queried metagenome. As demonstrated above, these containment scores can be used to identify sample contamination, recruit a set of reference genomes for metagenomic analysis, establish the absence of specific reference genomes in public databases, and retrospectively discover novel genomes.

As with other $k$-mer-based analyses, the containment estimate conflates sequence divergence with genome coverage. For example, a low-abundance species in a metagenome will typically receive a lower containment score than the true identity, simply because much of a query genome may not be fully represented by the sequencing reads. Similarly, short, high-identity regions of a genome, such as horizontally transferred elements, are not distinguished from weak, overall similarity. The downstream analyses we have performed in our experiments, such as mapping reads to discovered genomes, thus remain a crucial part of deploying Mash Screen in practice. It may be possible to disentangle these factors statistically using the multiplicity of the $k$-mers observed in the metagenome (e.g., as a proxy for sequence coverage). Conceptually, higher multiplicity for matching $k$-mers should indicate that $k$-mers not observed in the mixture are more likely to be missing due to sequence divergence, rather than low sequence coverage. Mash Screen currently tracks and reports the multiplicity of every hash in the reference sketches, but further work is needed to exploit this information fully.

Mash Screen is designed to efficiently estimate the containment of whole genomes or proteomes and can also be used to detect the presence of any set of related sequences (e.g., plasmids, functional pathways). However, the efficiency of MinHash is lost when the reference sequences are individual genes and proteins. In these cases, the sketch of a gene can be as large as the gene itself. As a sketch, by default, uses 8 bytes for each hash, a sequence would have to be roughly an order of magnitude larger in length than the sketch size (1000 by default) to accrue indexing storage benefits. Further, hashing $k$-mers in the input stream is a nontrivial component of the computational cost, and this step, which is important for probabilistic estimation, would not be necessary when simply counting all $k$-mers. While Mash will operate normally under these conditions, searching for short sequences within metagenomes is thus better left to traditional sequence search and mapping tools.

\section{Future directions}

In addition to searching for contamination, we also demonstrated the potential of using a containment screen to pre-filter a metagenome classification database. This would be especially useful for tools that rely on mapping or otherwise comparing metagenomic sequence reads to a large databases of reference genomes [27-29]. However, more rigorous validation of this use case is needed to prove its value. It may also be valuable to explore possible integration with classification pipelines to filter databases dynamically, given that database management is a burdensome step for many classification tools.

By definition of the MinHash process, similar reference genomes will share many minimum hashes between them. This makes the Mash Screen reference database highly compressible and keeps memory requirements low. In its current implementation, an off-the-shelf hash map is used for tracking reference sketch containment. As reference databases continue to grow, it may be desirable to improve the performance of this index. This could be achieved via a minimum perfect hash function for mapping $k$-mers to reference sketches, which would require only a few bits per $k$-mer to store the reference sketches [30].

\section{Summary}

Our online approach to containment estimation offers advantages over purely precomputed approaches, namely 
that Mash sketches can now be used for both genome resemblance and containment operations. We validated Mash Screen by correlating pairwise resemblance with containment and demonstrated its utility for contaminant screening and genome discovery. Further, we leveraged the efficiency of our method to compute the containment of all RefSeq genomes and plasmids within all SRA metagenomes, providing the research community with an unprecedented resource for retrospective data mining.

\section{Methods}

\section{Mathematical foundations}

Mash Screen compresses and indexes a database of reference sequences and measures their containment within a metagenome (the mixture). When estimating containment, all $k$-mers of the mixture are considered, ensuring adequate sensitivity. Because of this, any method for sampling the reference $k$-mer sets uniformly at random would suffice. However, for compatibility and to improve indexing efficiency, the reference sequences are sketched using the same bottom sketch strategy as Mash. Formally, a bottom sketch is defined as:

$$
S(A)=M I N_{m}(\pi(A)),
$$

where $\pi: \Omega \rightarrow \Omega$ is a min-wise independent permutation [31] (typically approximated with a hash function) and $\operatorname{MIN}_{m}(W)$ is the first $m$ elements of the ordered set $W$. Computing bottom sketches for the reference sequences maintains compatibility between the resemblance and containment operations of Mash, as well as improving the efficiency of indexing. Because bottom sketching is a form of locality sensitive hashing, the sketches of two similar sequences will contain many of the same hashes. This reduces the necessary index size for reference databases containing many similar sequences (e.g., strains of the same microbial species).

To estimate the containment index $c_{k}$ of a set of reference $k$-mers $A$ within some mixture $B$, we compare the sketch of $A$ against all of $B$ :

$$
c_{k}(a, b) \approx \frac{|S(A) \cap \pi(B)|}{|S(A)|}
$$

Since, for an arbitrary subset $A^{\prime} \subseteq A$, every element of $A^{\prime} \cap B$ must also be an element of $A \cap B$, it follows that if $A^{\prime}$ is chosen uniformly at random from $A, c_{k}(a, b)$ will be an unbiased estimate of the containment with expected error $O(1 / \sqrt{m})$.

Lastly, it is convenient to convert these set indices into estimates of sequence identity. Both the Jaccard index and the containment index drop exponentially with decreasing sequence identity. This is due to the fact that a single character substitution between two sequences affects $k k$ mers overlapping that position. In our prior work, $k$-mer survival was thus modeled as a Poisson process, yielding the Mash Distance:

$$
M_{D}(a, b)=-\frac{1}{k} \ln \frac{2 j}{1+j},
$$

where $j$ is the estimated Jaccard index. Similarly, we can estimate the identity of a genome and its assumed counterpart in a mixture, which we refer to as the Mash containment score $M_{c}(a, b)$. In this work, we adopt the binomial, which behaves more desirably in edge cases than its Poisson approximation, for modeling $k$-mer survival. Given two sequences of equal length with a sequence identity of $i_{a, b}$, and assuming $k$-mers are unique and independent, the probability that any $k$-mer in $A$ will be shared with $B$ is $i_{a, b}^{k}$. Because $c_{k}(a, b)$ represents the fraction of $k$ mers in $A$ shared with $B$, we can estimate this identity as:

$$
M_{c}(a, b)=i_{a, b} \approx c_{k}(a, b)^{\frac{1}{k}}
$$

Error bounds on this estimate for various values of $s$ and $k$ can be computed using the bounds subcommand. Note that, like the Mash Distance, this formula does not attempt to separate divergence from missing sequence. In cases where the mixture contains small pieces of a genome, but at high identity (e.g., potentially with horizontal transfer), we expect the Mash containment score to be closer to identity than coverage (Additional file 1: Figure S1).

\section{Amino acid estimation}

Mash Screen supports six-frame translation of reads against reference sketches of protein sequences. This is performed by including hash values for all six translations of each amino acid $k$-mer in the MinHash containment estimation (see "Streaming"). The out-of-frame elements are not expected to match $S(A)$ and will not affect the containment score since $c_{k}(a, b)$ is independent of the size of $B$. While very large read sets may cause some erroneously translated matches by chance, this is captured by a $p$ value, described below, that can be used to ensure only significant hits are reported.

\section{Assessing significance}

In MinHash resemblance computations, erroneous $k$ mers (i.e., those arising from sequencing error) can crowd correct $k$-mers out of sketches, reducing sensitivity. This is not an issue when screening a read set, since, if present, the correct $k$-mers will be observed regardless of any other $k$-mers that may contain errors. It is, however, important to consider the effects of such $k$-mers on specificity, given that the hash of an erroneous $k$-mer could coincidentally match that of a $k$-mer in the reference. This is especially true when performing six-frame translation, given that, lacking a priori knowledge of the correct reading frame, five spurious $k$-mers must be emitted for every true one, 
even in the absence of sequencing error. We address this by formulating a $p$ value to estimate the significance of a distance estimate given the background of the observed $k$-mer space. Because the containment index does not depend on the size of the larger set, the expected containment of one random set, $X$, within another, $Y$, is simply the probability $r$ that a random $k$-mer, $K$, appears in $Y$ :

$$
r=P(K \in Y)=\frac{n}{|\Sigma|^{k}}
$$

where $n$ is the number of distinct $k$-mers in $Y, \Sigma$ is the alphabet, and $k$ is the length of each $k$-mer. Thus, the probability that $Y$ matches at least $x$ out of $s k$-mers in the sketch of $X$ is given by the binomial distribution:

$$
p(x ; s ; r)=1-\sum_{i=0}^{x-1}\left(\begin{array}{l}
s \\
i
\end{array}\right) r^{i}(1-r)^{s-i} .
$$

For six-frame translation, the set size $n$ is based on all amino acid $k$-mers emitted, ensuring that the risk of false positives from saturation of the $k$-mer space is reflected in the $p$ value. It should be noted that this value does not consider multiple testing, but can be scaled by the number of sketches in the reference set to attain an expectation.

\section{Implementation}

Mash Screen is implemented in $\mathrm{C}++$ and is integrated into the existing Mash codebase as of v2.0. It is invoked as a subcommand, screen, similarly to dist, which is used computing the standard Mash distance. Unlike dist, however, screen must be given a pre-processed multi-sketch file to screen the reads against. This sketch file can be created with the sketch command in the same manner as for dist. For compatibility with existing Mash sketches, MurmurHash3 [32] is used to hash $k$-mers, as in Ondov et al. [7]. If the sketch is created from protein sequences, the reads given to screen will automatically be translated into all six frames during screening.

\section{Streaming}

As the mixture is intended to be an arbitrarily large set, such as a sequencing run, it is not desirable to load the entire set into memory. In contrast, the reference sketches are small and can easily be indexed in memory. To compute the containment of each reference sketch, the mixture is streamed against this index (Fig. 2). First, all distinct hashes present in the constituent sketches are collected into a reference set. This set is used as the keys to a map (implemented here as unordered map in the $\mathrm{C}++$ Standard Template Library), where the associated values are counts for how many times the hash appears in the mixture, set initially to zero. Sequences from the mixture are then streamed, translating to six frames if applicable, and all their $k$-mers hashed, incrementing the counters in the map if a key for that hash is present. This process is parallelized by using atomic operations to increment to the counters without locking the map. Finally, a distance for each constituent is determined by tallying how many hashes in its sketch have non-zero counts in the map. Note that the number of entries in this map, and thus memory used, will depend on the number of distinct hashes in the complete set of possible constituents. Since many reference genomes will be highly similar to one another, storing a MinHash sketch for each reference is more efficient than storing a random selection of $k$-mers (as noted above). For example, in the experiments above, the collection of RefSeq sketches contained only $1.97 \times 10^{7}$ distinct hashes in nucleotide space $(k=21)$ and $1.36 \times 10^{7}$ in amino acid space $(k=9)$, compared to the $1.27 \times 10^{8}$ total (independent of $k$ and alphabet) when the sketch size is 1000 .

\section{$P$-value}

The $p$ value (described in Eq. 6), used to estimate the significance of a given distance estimate, relies on the number of distinct $k$-mers $|B|$ observed in the streamed reference set. Counting this number exactly would require an entry for each of these $k$-mers in memory, negating the memory efficiency afforded by the streaming estimation algorithm. Instead, this count is estimated from the maximum hash value of an auxiliary MinHash sketch of the reference set, constructed as the set is streamed for this purpose [33]. If the sketch size is $s$, its maximum hash value is $v$, and each hash uses $b$ bits, the number of distinct $k$-mers in the genome is estimated as $n=2^{b} \frac{s}{v}$.

\section{Experiments \\ Correlation between Mash distance and containment estimates}

Simulated reads were generated with ART [34] version "Mount Rainier" using the art illumina command with parameters -f 50 (50--fold coverage), - I 100 (100-base read length), and -ss HS20 (Illumina HiSeq 2000 platform profile), and with the combined 64 reference genomes as input. Mash was run with defaults for both screen and dist, except the use of $-p$ for multiple threads. RefSeq genomes were binned to Shakya reference genomes with a custom script. Mash Screen (v2.1.1) was run with 32 threads $(-\mathrm{p} 32)$ on a workstation with dual, $2.20 \mathrm{GHz}, 20$-core, hyperthreaded CPUs. The query was a sketch of RefSeq release 93 built wth the default $k$ (21), the default sketch size (1000), and a seed of $0\left(\begin{array}{ll}-S & 0\end{array}\right)$.

\section{Correlation of Mash Screen containment scores with DIAMOND read mapping identity}

Blast was run with default parameters (using the blastp command), a custom script was used to collect identifiers of best hits, and blastdbcmd was used to extract these 
sequences from $n r$. The diamond makedb subcommand was run with defaults. The diamond blastx command was run with --more-sensitive, --masking 0 (do not mask low-complexity regions), --threads 32, and --outfmt 101 (SAM format). The SamTools [35] mpileup command was used to create pileups from the DIAMOND mappings, and a custom script was used to determine consensus identity and coverage from the pileups. Mash Screen v2.2 was used for this experiment. The query was a sketch of the protein set built with defaults for amino acids ( $k=9$, sketch size 1000).

\section{Screening all metagenomes in the Sequence Read Archive}

Jobs were run on nodes of the Biowulf cluster, using Mash Screen v2.0. configurations SRA accessions for runs labeled as metagenomic were extracted with Bioconductor [36]. Reads for each run were streamed to an instance of mash screen using fastq-dump from the SRA Toolkit [4] with the $-\mathrm{Z}$ flag. Default options were used for mash screen except -p 2 to specify 2 threads. Results for each run were filtered and compiled into tables with custom scripts.

\section{Novel virus assembly}

The SRA project SRP064400 [37] was downloaded using fastq-dump from the SRA Toolkit [4], and assembled using metaSPAdes [38] with default parameters. Assembled contigs were screened using blastx and a custom blast database containing a select set of conserved proteins from DNA viruses [39]. Contigs containing likely viral protein sequences were further screened against all known viruses using blastn. Contigs with less than 90\% nucleotide identity to any known viral genome were then manually annotated and analyzed for completeness, which revealed a nearly complete genome for a new polyomavirus related to HPyV6 and HPyV7. The viral genome was completed using methods previously described by Pastrana and colleagues [39].

\section{Supplementary information}

Supplementary information accompanies this paper at https://doi.org/10.1186/s13059-019-1841-x.

Additional file 1: Figure S1. The results of experiments on limits of Mash Screen detection.

Additional file 2: Review history.

\section{Peer review information}

Kevin Pang was the primary handling editor on this article and managed its editorial process and peer review in collaboration with the rest of the editorial team.

\section{Acknowledgements}

We thank Brian Walenz for helpful discussions about implementation details. This research utilized the computational resources of the NIH HPC Biowulf cluster (https://hpc.nih.gov).

\section{Authors' contributions}

The screening algorithm was designed by BDO and AMP and implemented by BDO. Validation using the Shakya metagenome was performed by BDO. SRA experiments were performed by BDO and AK. MinHash protein space experiments were performed by BDO and AS. Viral discovery, assembly, and analysis were performed by GJS, CBB, AK, and AMP. Classification database reduction experiments were performed by BDO and SK. The study was conceived and coordinated by AMP. All authors read and approved of the final text.

\section{Funding}

This research was supported in part by the Intramural Research Programs of the National Human Genome Research Institute and the National Cancer Institute, National Institutes of Health.

\section{Availability of data and materials}

Mash is open source and distributed under a BSD license at https://github. $\mathrm{com} / \mathrm{marbl} / \mathrm{mash}$ [40]. This study made extensive use of public datasets available from the Sequence Read Archive (SRA) [4] and, in particular, performed targeted experiments using SRP064400 [37], SRS015937 [21], SRS02026 [21], SRR606249 [17], and ERR024951 [41]. The datasets generated and/or analyzed during the current study are available at https://mash. readthedocs.io/en/latest/data.html.

\section{Ethics approval and consent to participate}

Not applicable.

\section{Consent for publication}

Not applicable.

\section{Competing interests}

The authors declare that they have no competing interests.

\section{Author details}

${ }^{1}$ Genome Informatics section, National Human Genome Research Institute, Bethesda, MD, USA. ${ }^{2}$ Department of Computer Science, University of Maryland College Park, College Park, MD, USA. ${ }^{3}$ Tumor Virus Molecular Biology section, National Cancer Institute, Bethesda, MD, USA. ${ }^{4}$ Department of Electrical Engineering and Computer Science, Massachusetts Institute of Technology, Cambridge, MA, USA. ${ }^{5}$ Department of Computer Science, Princeton University, Princeton, NJ, USA.

Received: 27 February 2019 Accepted: 27 September 2019

Published online: 05 November 2019

\section{References}

1. O'Leary NA, Wright MW, Brister JR, Ciufo S, Haddad D, McVeigh R, Rajput B, Robbertse B, Smith-White B, Ako-Adjei D, et al. Reference sequence (RefSeq) database at NCBI: current status, taxonomic expansion, and functional annotation. Nucleic Acids Res. 2015;44(D1):733-45.

2. RefSeq growth statistics. https://www.ncbi.nlm.nih.gov/genbank/ statistics/. Accessed 27 Feb 2019.

3. GenBank and WGS Statistics. http://www.ncbi.nlm.nih.gov/genbank/. Accessed 27 Feb 2019.

4. Leinonen $R$, Sugawara $H$, Shumway M, International Nucleotide Sequence Database Collaboration. The sequence read archive. Nucleic Acids Res. 2011;39(Database issue):19-21.

5. SRA database growth. https://www.ncbi.nlm.nih.gov/sra/docs/ sragrowth/. Accessed 27 Feb 2019.

6. Stephens ZD, Lee SY, Faghri F, Campbell RH, Zhai C, Efron MJ, lyer R, Schatz MC, Sinha S, Robinson GE. Big data: astronomical or genomical? PLoS Biol. 2015;13(7):1002195.

7. Ondov BD, Treangen TJ, Melsted P, Mallonee AB, Bergman NH, Koren S, Phillippy AM. Mash: fast genome and metagenome distance estimation using minhash. Genome Biol. 2016;17(1):132.

8. Broder AZ. On the resemblance and containment of documents. In: Compression and Complexity of Sequences 1997. Proceedings; 1997. p. 21-9. https://doi.org/10.1109/sequen.1997.666900.

9. Marchet C, Lecompte L, Limasset A, Bittner L, Peterlongo P. A resource-frugal probabilistic dictionary and applications in bioinformatics. Discret Appl Math. 2018. https://doi.org/10.1016/j.dam.2018.03.035. 
10. Shrivastava A. Optimal densification for fast and accurate minwise hashing. arXiv preprint. 2017. arXiv:1703.04664.

11. Zhao X. Bindash, software for fast genome distance estimation on a typical personal laptop. Bioinformatics. 2018. https://doi.org/10.1093/ bioinformatics/bty651.

12. Baker DN, Langmead B. Dashing: Fast and accurate genomic distances with hyperloglog. BioRxiv. 2018;501726:. https://doi.org/10.1101/501726.

13. Koslicki D, Zabeti H. Improving minhash via the containment index with applications to metagenomic analysis. Appl Math Comput. 2019;354: 206-15.

14. Solomon B, Kingsford C. Fast search of thousands of short-read sequencing experiments. Nat Biotechnol. 2016;34(3):300.

15. Pandey P, Almodaresi F, Bender MA, Ferdman M, Johnson R, Patro R. Mantis: a fast, small, and exact large-scale sequence-search index. Cell Syst. 2018;7(2):201-7

16. Bradley P, den Bakker HC, Rocha EP, McVean G, lqbal Z. Ultrafast search of all deposited bacterial and viral genomic data. Nat Biotechnol. 2019;37(2):152.

17. Shakya M, Quince C, Campbell JH, Yang ZK, Schadt CW, Podar M. Comparative metagenomic and rrna microbial diversity characterization using archaeal and bacterial synthetic communities. Environ Microbiol. 2013;15(6):1882-99.

18. Awad S, Irber L, Brown CT. Evaluating metagenome assembly on a simple defined community with many strain variants. bioRxiv. 2017;155358:. https://doi.org/10.1101/155358.

19. Kook J-K, Park S-N, Lim YK, Cho E, Jo E, Roh H, Shin Y, Paek J, Kim H-S, Kim H, et al. Genome-based reclassification of fusobacterium nucleatum subspecies at the species level. Curr Microbiol. 2017;74(10):1137-47.

20. Buchfink B, Xie C, Huson DH. Fast and sensitive protein alignment using diamond. Nat Methods. 2014;12(1):59.

21. Lloyd-Price J, Mahurkar A, Rahnavard G, Crabtree J, Orvis J, Hall AB, Brady A, Creasy HH, McCracken C, Giglio MG, et al. Strains, functions and dynamics in the expanded Human Microbiome Project. Nature. 2017;550(7674):61.

22. Kamminga S, van der Meijden E, Wunderink HF, Touzé A, Zaaijer HL, Feltkamp MC. Development and evaluation of a broad bead-based multiplex immunoassay to measure lgG seroreactivity against human polyomaviruses. J Clin Microbiol. 2018;56(4):01566-17.

23. Kamminga S, van der Meijden E, Feltkamp MC, Zaaijer HL. Seroprevalence of fourteen human polyomaviruses determined in blood donors. PloS ONE. 2018;13(10):0206273.

24. Buck CB, Van Doorslaer K, Peretti A, Geoghegan EM, Tisza MJ, An P, Katz JP, Pipas JM, McBride AA, Camus AC, et al. The ancient evolutionary history of polyomaviruses. PLoS Pathog. 2016;12(4):1005574.

25. Gedvilaite A, Tryland M, Ulrich RG, Schneider J, Kurmauskaite V, Moens $U$, Preugschas H, Calvignac-Spencer S, Ehlers B. Novel polyomaviruses in shrews (Soricidae) with close similarity to human polyomavirus 12. J Gen Virol. 2017;98(12):3060-7.

26. Mishra N, Pereira M, Rhodes RH, An P, Pipas JM, Jain K, Kapoor A, Briese $T$, Faust PL, Lipkin WI. Identification of a novel polyomavirus in a pancreatic transplant recipient with retinal blindness and vasculitic myopathy. J Infect Dis. 2014;210(10):1595-9.

27. Wood DE, Salzberg SL. Kraken: ultrafast metagenomic sequence classification using exact alignments. Genome Biol. 2014;15(3):46.

28. Kim D, Song L, Breitwieser FP, Salzberg SL. Centrifuge: rapid and sensitive classification of metagenomic sequences. Genome Res. 2016;26(12):1721-9.

29. Dilthey A, Jain C, Koren S, Phillippy A. Metamaps-strain-level metagenomic assignment and compositional estimation for long reads. BioRxiv. 2018;372474:. https://doi.org/10.1101/372474.

30. Limasset A, Rizk G, Chikhi R, Peterlongo P. Fast and scalable minimal perfect hashing for massive key sets. In: 16th International Symposium on Experimental Algorithms. Dagstuhl: Schloss Dagstuhl; 2017. p. 1-11.

31. Broder AZ, Charikar M, Frieze AM, Mitzenmacher M. Min-wise independent permutations. J Comput Syst Sci. 2000;60(3):630-59.

32. MurmurHash3. https://code.google.com/p/smhasher. Accessed 20 Mar 2015.

33. Bar-Yossef Z, Jayram T, Kumar R, Sivakumar D, Trevisan L. Counting distinct elements in a data stream. In: International Workshop on Randomization and Approximation Techniques in Computer Science. Springer; 2002. p. 1-10. https://doi.org/10.1007/3-540-45726-7_1.
34. Huang W, Li L, Myers JR, Marth GT. Art: a next-generation sequencing read simulator. Bioinformatics. 2011;28(4):593-4.

35. Li H, Handsaker B, Wysoker A, Fennell T, Ruan J, Homer N, Marth G, Abecasis G, Durbin R. The sequence alignment/map format and samtools. Bioinformatics. 2009;25(16):2078-9.

36. Gentleman RC, Carey VJ, Bates DM, Bolstad B, Dettling M, Dudoit S, Ellis B, Gautier L, Ge Y, Gentry J, et al. Bioconductor: open software development for computational biology and bioinformatics. Genome Biol. 2004;5(10):80.

37. Vincent C, Miller MA, Edens TJ, Mehrotra S, Dewar K, Manges AR. Bloom and bust: intestinal microbiota dynamics in response to hospital exposures and clostridium difficile colonization or infection. Microbiome. 2016;4(1):12.

38. Nurk S, Meleshko D, Korobeynikov A, Pevzner PA. metaSPAdes: a new versatile metagenomic assembler. Genome Res. 2017;213959:. https://doi. org/10.1101/gr.213959.116.

39. Pastrana DV, Peretti A, Welch NL, Borgogna C, Olivero C, Badolato R, Notarangelo LD, Gariglio M, FitzGerald PC, McIntosh CE, et al. Metagenomic discovery of 83 new human papillomavirus types in patients with immunodeficiency. mSphere. 2018;3(6):00645-18.

40. Ondov B, Koren S, Treangen T, Sappington A, Melsted P, Phillippy A. Mash. 2019. https://doi.org/10.5281/zenodo.3461012. https://doi.org/10. 5281/zenodo.3461012.

41. Petrovska L, Mather AE, AbuOun M, Branchu P, Harris SR, Connor T, Hopkins KL, Underwood A, Lettini AA, Page A, et al. Microevolution of monophasic Salmonella typhimurium during epidemic, United Kingdom, 2005-2010. Emerg Infect Dis. 2016;22(4):617.

\section{Publisher's Note}

Springer Nature remains neutral with regard to jurisdictional claims in published maps and institutional affiliations.

Ready to submit your research? Choose BMC and benefit from

- fast, convenient online submission

- thorough peer review by experienced researchers in your field

- rapid publication on acceptance

- support for research data, including large and complex data types

- gold Open Access which fosters wider collaboration and increased citations

- maximum visibility for your research: over $100 \mathrm{M}$ website views per year

At $\mathrm{BMC}$, research is always in progress.

Learn more biomedcentral.com/submissions 